\title{
Fluorescence alteration of oil inclusions under ultraviolet irradiation
} Key Laboratory of Tectonics and Petroleum Resources of Ministry of Education, China University of Geosciences, Wuhan 430074, China;
*Corresponding author, E-mail: tiger_lcq@sina.com

(Received: January 12, 2021; Revised accepted: May 23, 2021)

https://doi.org/10.18814/epiiugs/2021/021009

The fluorescence colors of oil inclusions can be influenced by many geological factors including thermal maturity. However, the ultraviolet (UV) irradiation during laboratory process can also induce a significant alteration in fluorescence spectra of oil inclusions, which is seldom considered when using fluorescence to classify oil inclusions. Four oil inclusions from the Maokou Formation in the central Sichuan Basin, China were performed continuous UV irradiation. Their fluorescence spectra were recorded over time. The vapor / liquid phase ratio was measured by a confocal laser scanning microscope (CLSM). The results reveal a generally irreversible alteration of oil inclusion fluorescence spectra. $\lambda_{\max }$ shows long-time stable and instant mutation, whereas $Q F-535$ and $Q_{650 / 500}$ exhibit monotone increasing trend. Recorded $\lambda_{\max }$ can represent the original based on spectral shape analysis, however, recorded $Q F-$ 535 and $Q_{650 / 500}$ require a regression model to predict original values. Besides, there is a potential change in vapor / liquid ratio of oil inclusion after long and intense UV irradiation. The conclusion is that the thermal maturity determination by fluorescence spectra of oil inclusions should be used with care, because the spectra are easily altered during petrography study before being recorded.

\section{Introduction}

Fluid inclusions are generally entrapped during mineral crystallization (primary) or during healing of fractures (secondary) (Goldstein, 2001). As a sealed capsule, the fluids in fluid inclusions can preserve the geofluids environment (temperature, pressure, composition, etc.) when they were entrapped, if significant re-equilibration mechanism on these fluid inclusions can be eliminated (Goldstein, 2001). Hydrocarbon-bearing fluid inclusions were, therefore, widely used by petroleum geologists working for oil companies to investigate hydrocarbon evolution history from its generation to migration to accumulation in worldwide petroliferous basins. Oil inclusions can easily be separated from aqueous inclusions by their fluorescence under ultraviolet (UV) excitation. Fluorescence of oil is related to energy transition from a ground state energy level to an excited state energy level in the $\pi$ orbitals $(\mathrm{C}=\mathrm{C})$ in aromatic and polyaromatic compounds. The fluorescence emission shifts to longer wavelength as the number of aromatic rings in the molecule increases. NSO compounds show a less intensive fluorescence at longer wavelength than the aromatic compounds. Therefore, the fluorescence spectra are used to classify oil inclusions, especially to estimate their thermal maturities. Fluorescence spectral parameters have been proposed, including $\lambda_{\max }$ (wavelength of maximum intensity), QF-535 (ratio of the 535-750 nm flux to the $430-535 \mathrm{~nm}$ flux), and $\mathrm{Q}_{650 / 500}$ (ratio of the intensity at $650 \mathrm{~nm}$ to the intensity at $500 \mathrm{~nm}$ ), to quantitatively describe the fluorescence spectra of oil inclusions (Munz, 2001). Stasiuk and Snowdon (1997) established a positive correlation between $\lambda_{\max }-\mathrm{Q}_{650 / 500}$ and thermal maturity parameters $\mathrm{nC}_{17}$ /pristane and $\mathrm{nC}_{18}$ /phytane. Fluorescence color changes from red to yellow to green to blue (blue shift) as the thermal maturity of oil increases, and $\lambda_{\max }, \mathrm{QF}-535$ and $\mathrm{Q}_{650 / 500}$ decrease correspondingly. However, it has been proved that these simply acquired fluorescence parameters cannot be directly used to estimate thermal maturity of oil inclusions. In spite of thermal maturity, some geological processes, including source, water washing and trapping fractionation can also significantly influence fluorescence color (George et al., 2001). Some recent studies laid constraints on the relationship between fluorescence color and thermal maturity of oil inclusions that a blue shift for higher maturity is valid only when the source rocks reach the main oil generation and expulsion stage (Ping et al., 2019a). The blue fluorescence color of an oil inclusion is more likely to be shifted from a previous yellow or green fluorescence due to secondary cracking process instead of from high maturity oil directly generated from source rocks (Ping et al., 2019b). The geological processes induced fluorescence alteration against the thermal maturity can be identified by molecular analysis of inclusion oils by following standard procedures (George et al., 2007).

Unfortunately, the fluorescence spectra do not seem as accurate as they were discussed in many researches even if the secondary alteration caused by geological processes could be eliminated, because the fluorescence of oil inclusions can also be altered by prolonged exposure under UV light (Pironon and Pradier, 1992). As shown in the experiment, the fluorescence intensity and QF-535 change monotonously (increase or decrease) with time of exposure under UV. The fluorescence parameters change rapidly in the first $2 \mathrm{~min}$ and slow down 
after that (Pironon and Pradier, 1992). This phenomenon is explained to be related to the formation of aryl-ethers for the positive alteration (increase) and the appearance of unconjugated carbonyl groups for the negative alteration (decrease) (Pradier et al., 1990). Chemical composition of oil inclusion changes under UV and so do fluorescence spectra parameters. These parameters changed significantly within a very short time interval ( $2 \mathrm{~min})$, which is shorter than the time that petrography study usually needs. Therefore, whether the fluorescence spectra obtained after detailed petrography study of oil inclusions can represent the initial oil inclusion fluorescent properties remains a problem. Except the geological processes that alter the fluorescence color, the experimental UV exposure also should be seriously considered, and if possible, would this experimental factor be eliminated?

The main objectives of this study are to 1) find out the influence of UV irradiation on fluorescence spectra of oil inclusions, 2) discuss the validity of fluorescence spectra of oil inclusions recorded in laboratory routine, 3 ) propose a preliminary method to predict the original spectral parameters after a significant alteration by UV irradiation, and 4) discuss the reversibility of fluorescence analysis on oil inclusions.

\section{Sample and Experiments}

One dolomite hand sample was collected from the Permian Maokou Formation in the central part of the Sichuan Basin, southwestern China. The burial depth of the sample is $4705.28 \mathrm{~m}$.

Oil inclusions were identified on doubly polished thin sections using a Nikon 80I dual-channel fluorescence microscope equipped with two objective lenses $(20 \times$ and $50 \times)$. Characterization of oil inclusion were examined in incident fluorescence light mode and transmitted light (TR) mode. UV was provided by a $100 \mathrm{~W}$ mercury lamp with excitation at 330-380 nm. A Nikon filter UV-2A (EX 330-380, DM 400, BA 420) was used. The fluorescence spectra were recorded using a Maya 2000 Pro spectrometer and processed with the Yuanao spectroscopy system at room temperature. The signal accumulation time was 200 milliseconds $(0.2 \mathrm{~s})$. Each spectrum was averaged from 20 continuous recorded spectra. The fluorescence of host minerals (dolomite) was eliminated at the beginning of each document series by a subduction of the fluorescence signal of the host minerals from the software.

Fluorescence spectra of four oil inclusions (OI1, OI2, OI3 and OI4), respectively exhibiting bule, blue, blue and yellow fluorescence colors, were recorded through $20 \times$ lens right after the petrography study. The $50 \times$ lens was not used during this process because the four oil inclusions can surely be illuminated by UV at the same time under $20 \times$ lens. After that, the liquid phase volume of the two-phase oil inclusion (OI1) was measured carefully by using a Leica SPE DM5500 confocal laser scanning microscope (CLSM) at room temperature. Here, only OI1 was selected for CLSM because it is the only twophase oil inclusion (Fig. 1). A week later, these four oil inclusions were exposed to a constant UV irradiation condition as previously described. Because the CLSM analysis was performed on OI1 after the initial fluorescence spectra measurements, the fluorescence spectrum of OI1 was considered to be significantly altered by the intense UV irradiation of CLSM. The alteration was determined by a spectrum of OI1 recorded at the beginning ( 0 minute) of the UV irradiation experiment (Fig. 2B). At this time, the other three oil inclusions were also exposed to constant UV irradiation, thus, only the fluorescence spectrum of OI 1 was recorded every 5 minutes from 0 to 210 minute. To avoid the environmental variation, the sample was not moved during the experiment and the fluorescence signal reception position was fixed. After a 210-minute UV irradiation, the fluorescence spectra of these four oil inclusions were measured again (Fig. 2C). The comparison of the final spectra $(210 \mathrm{~m})$ to the initial spectra $(0 \mathrm{~m})$ can indicate the fluorescence alteration caused by long-time UV irradiation. OI2, OI3 and OI4 were not measured spectra at the beginning of the experiment $(0$ $\mathrm{m}$ ), however, their initial spectra (Fig. 2A) can be considered as the started spectra as they were not performed CLSM analysis which may significantly alter the fluorescence spectra before the UV irradiation experiment.

To understand the variations of fluorescence spectra of other three oil inclusions as well as to see if there is any reversible change and to have an analogue, UV fluorescence spectra of these four oil inclusions were recorded after a 24-hour-break and recorded after another 24hour-break (48 hours later since the 0-210-minute experiment) with a step of 5 minutes, respectively. Being this a preliminary study, we did not know if the time dependent changes of fluorescence spectra were reproducible, thus, more precise measurement on oil inclusion OI1 was performed after 6 hours ( 54 hours after the 0-210-minute experiment). The fluorescence spectrum of OI 1 was recorded by a step of $30 \mathrm{~s}$ from 0 to $5 \mathrm{~m}$. All the UV fluorescence spectra measurements were controlled in the same condition. After that, CLSM analysis was conducted again on oil inclusion OI1 to determine if any vapor phase alteration was caused by UV exposure. The two runs of CLSM analysis on OI1 were kept in the same analytical condition to avoid any man-made change. Also, the vapor bubble in OI1 did not move, indicating high viscosity, which allows the repeating CLSM analysis.

Five wavelength parameters were proposed as $\lambda_{\mathrm{b}}, \lambda_{\mathrm{c}}, \lambda_{\mathrm{g}}, \lambda_{\mathrm{y}}$ and $\lambda_{\mathrm{r}}$ in this study to better characterize the spectra. They represent the wavelengths of five peaks or potential peaks on the spectra respectively. $\lambda_{b}$ represents wavelength of around $455 \mathrm{~nm}$, corresponding to blue fluorescence; $\lambda_{\mathrm{c}}$ represents wavelength of around $492 \mathrm{~nm}$, corresponding to cyan fluorescence; $\lambda_{\mathrm{g}}$ represents wavelength of around $547 \mathrm{~nm}$, corresponding to green fluorescence; $\lambda_{y}$ represents wavelength of around $577 \mathrm{~nm}$, corresponding to yellow fluorescence; $\lambda_{\mathrm{r}}$ represents wavelength of around $633 \mathrm{~nm}$, corresponding to red fluorescence.

\section{Results}

\section{Fluid Inclusion Petrography}

Many oil inclusions were detected in dolomite crystals in our sample, but only four were selected for spectra analysis due to their large sizes $(10-30 \mu \mathrm{m})$ compared to others. The four oil inclusions are denoted as OI1, OI2, OI3 and OI4. During the petrography observation process, the OI1, OI2 and OI3 yielded blue fluorescence (Figs. $1 \mathrm{~B}$ and 1D), whereas OI4 yielded yellow fluorescence (Fig. 1F). Only OI1 is a two-phase oil inclusion (Figs. 1A and 1B), whereas OI2, OI3 and OI4 are all liquid phase at room temperature, which make them 


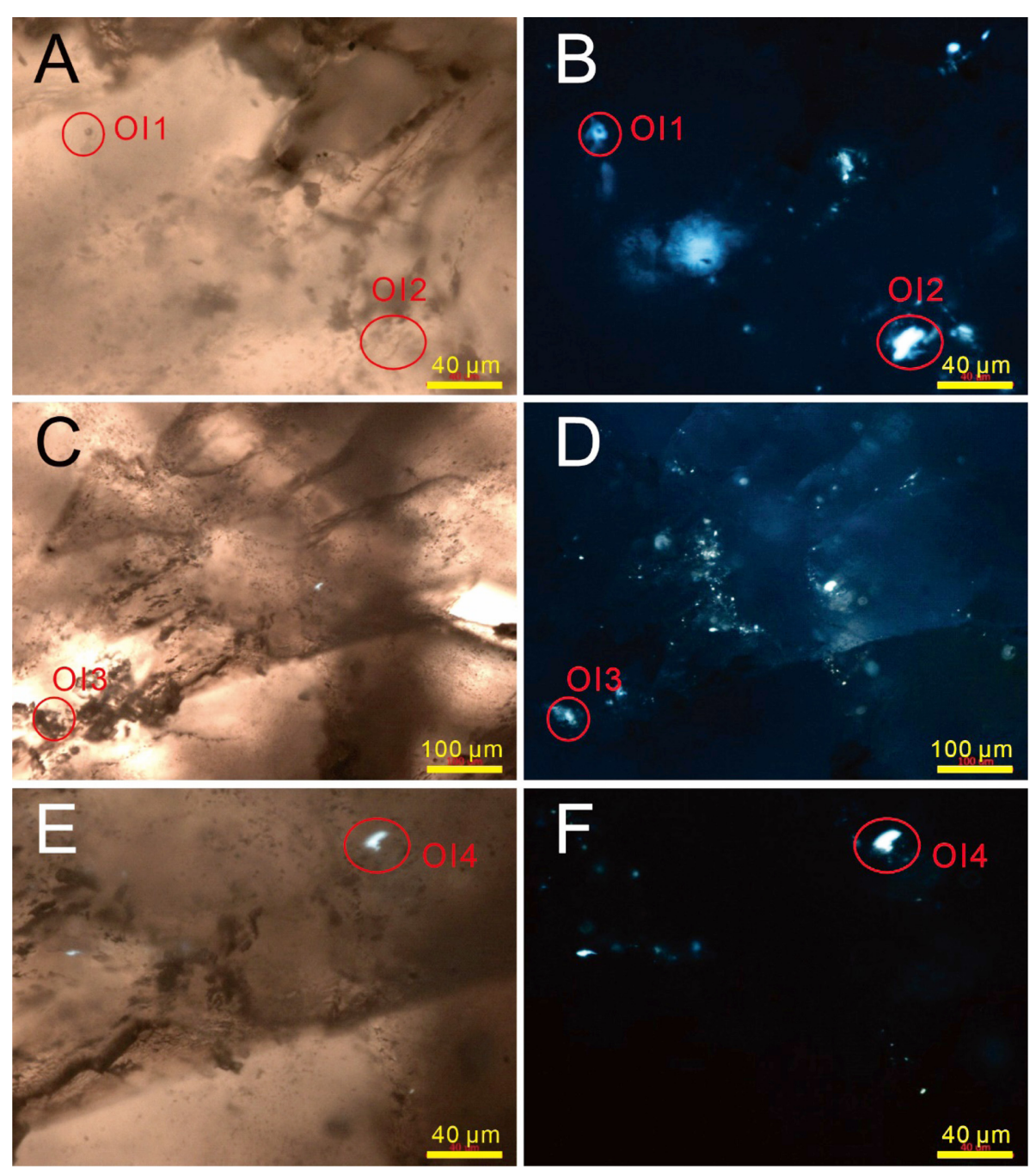

Figure 1. Photographs of oil inclusions in the dolomite crystals. (A) TR, OII and OI2; (B) UV, OII and $O I 2$ with blue fluorescence; $(C) T R$ and $U V, O I 3$; (D) $U V$, OI3 with blue fluorescence; $€ T R$ and $U V, O I 4 ;(F) U V, O I 4$ with yellow fluorescence.

difficult to be identified under TR. In this case, the photos of OI3 and OI4 under TR was combined with UV illumination for a better location (Figs. $1 \mathrm{C}$ and $1 \mathrm{E})$.

\section{Initial Fluorescence Spectra}

The initial recorded spectra of the four oil inclusions are shown in Fig. 2A, the corresponding standard fluorescence spectral parameters $\left(\lambda_{\max }, \mathrm{Q}_{650 / 500}\right.$ and QF-535) are listed in Table 1. These oil inclusions can be divided into two types based on fluorescence color as well as on spectral parameters. The type I oil inclusions (OI1, OI2, and OI3) are blue fluorescence with $\lambda_{\max }$ in a range of 456 and $460 \mathrm{~nm}, \mathrm{Q}_{650 / 500}$ from 0.36 to 0.44 and QF-535 between 0.87 and 1.05. Type II oil inclusion (OI4) is yellow fluorescence with $\lambda_{\max }$ of $577 \mathrm{~nm}, \mathrm{Q}_{650 / 500}$ of 0.68 and QF-535 of 1.57. In the spectra of type I oil inclusions, the relative intensity of $\lambda_{\mathrm{b}}, \lambda_{\mathrm{c}}, \lambda_{\mathrm{g}}, \lambda_{\mathrm{y}}$ and $\lambda_{\mathrm{r}}$ is $\lambda_{\mathrm{b}}>\lambda_{\mathrm{c}}>\lambda_{\mathrm{g}}>\lambda_{\mathrm{y}}>\lambda_{\mathrm{r}}$, whereas that in the spectrum of type II oil inclusion is $\lambda_{\mathrm{r}}<\lambda_{\mathrm{b}}<\lambda_{\mathrm{c}}<\lambda_{\mathrm{g}}<\lambda_{\mathrm{y}}$ (Fig. $2 \mathrm{~A})$. In type I oil inclusions, OI1 varies from OI 2 and OI 3 in the relative intensities of $\lambda_{\mathrm{c}}, \lambda_{\mathrm{g}}, \lambda_{\mathrm{y}}$, and $\lambda_{\mathrm{r}}$, in which those of OI $1<\mathrm{OI} 2<\mathrm{OI} 3$ (Fig. 2A).

\section{Temporal Fluorescence Spectra Alter- ation under UV Irradiation}

Four typical spectra of OI1 during the experiment were chosen to display on Fig. 2B. Compared to the initial spectrum of OI1, the spectrum at $0 \mathrm{~m}$ shows a decrease in the intensity of $\lambda_{\mathrm{b}}$ relative to $\lambda_{\mathrm{c}}$ and an increase in the intensities of $\lambda_{\mathrm{g}}, \lambda_{\mathrm{y}}$ and $\lambda_{\mathrm{r}}$ relative to $\lambda_{\mathrm{c}}$. As the UV irradiation continues, the alteration of spectrum continues and so as the relative intensities of $\lambda_{b}, \lambda_{c}, \lambda_{g}, \lambda_{y}$ and $\lambda_{b}$. The relative intensities of $\lambda_{\mathrm{b}}$ and $\lambda_{\mathrm{c}}$ decrease while those of $\lambda_{\mathrm{g}}, \lambda_{\mathrm{y}}$ and $\lambda_{b}$ increase (Fig. 2B). The alteration path of standard fluorescence spectral parameters $\left(\lambda_{\max }\right.$, $\mathrm{Q}_{650500}$ and QF-535) are shown in Fig. 3. Q650/500 and QF-535 both show positive alterations with UV irradiation time, however, $\lambda_{\max }$ shows a small increasement from 0 to $5 \mathrm{~m}$ and nearly stays constant from 5 to $95 \mathrm{~m}$ (Fig. 3). A mutation occurs at $100 \mathrm{~m}$, where $\lambda_{\max }$ changes from 498 to $579 \mathrm{~nm}$. Q650/500 and QF-535 alteration paths do not exhibit any obvious change at this time. The mutation of $\lambda_{\max }$ at $100 \mathrm{~m}$ is considered to be due to the gradually increasing intensity of $\lambda_{y}$ relative to the intensity of $\lambda_{c}$. The intensity of $\lambda_{y}$ finally exceeds the intensity of $\lambda_{\mathrm{c}}$ at $100 \mathrm{~m}$, as a consequence, $\lambda_{\max }$ changes from $\lambda_{c}$ to $\lambda_{y}$. After $100 \mathrm{~m}$, the previously smooth alteration paths show some little fluctuations. $\mathrm{Q}_{650 / 500}$ and QF-535 still exhibit positive alteration while $\lambda_{\max }$ continues to stay constant (Fig. 3). After a 210-minute UV irradiation, the spectrum of OI1 was completely altered. A spectrum of blue fluorescent oil inclusion finally changed to a spectrum of yellow fluorescent oil inclusion by long-time UV irradiation (Fig. 2B). One difference between the spectrum of OI1 at $210 \mathrm{~m}$ and the initial spectrum of OI4 is that the relatively high intensity of $\lambda_{\mathrm{r}}$ in OI1 (Figs. 2A and 2B).

The spectra of OI2, OI3 and OI4 at $210 \mathrm{~m}$ were also recorded, and together with that of OI1 are shown in Fig. 2C. Compared to the spectra at $0 \mathrm{~m}$ (OI1: $0 \mathrm{~m}$, others: initial), the $\mathrm{UV}$ irradiation causes a decrease in intensities of $\lambda_{\mathrm{b}}, \lambda_{\mathrm{c}}$ and $\lambda_{\mathrm{g}}$ relative to $\lambda_{\mathrm{y}}$, and an increase in intensity of $\lambda_{\mathrm{r}}$ relative to $\lambda_{\mathrm{y}}$ in both types of oil inclusions (Fig. $2 \mathrm{C}$ ). The difference is that these alterations completely change the spectra shapes and spectral parameters of type I oil inclusions, making blue fluorescence oil inclusion spectra become yellow fluorescent oil inclusion spectra ( $\lambda_{\max }$ changed), but have little influence on type II oil inclusion $\left(\lambda_{\max }\right.$ not changed). Standard spectral parameter values of spectra at $210 \mathrm{~m}$ are listed in Table 1. Except the $\lambda_{\max }$ of OI4 shows a small red shift ( $<$ $10 \mathrm{~nm}$ ) which can be treated as constant, the $\lambda_{\max }$ of type 1 oil inclusion and the $\mathrm{Q}_{650 / 500}$ and QF-535 of both types of oil inclusions all show significant increasements, indicating a red shift of fluorescence spectra will occur under UV irradiation. 

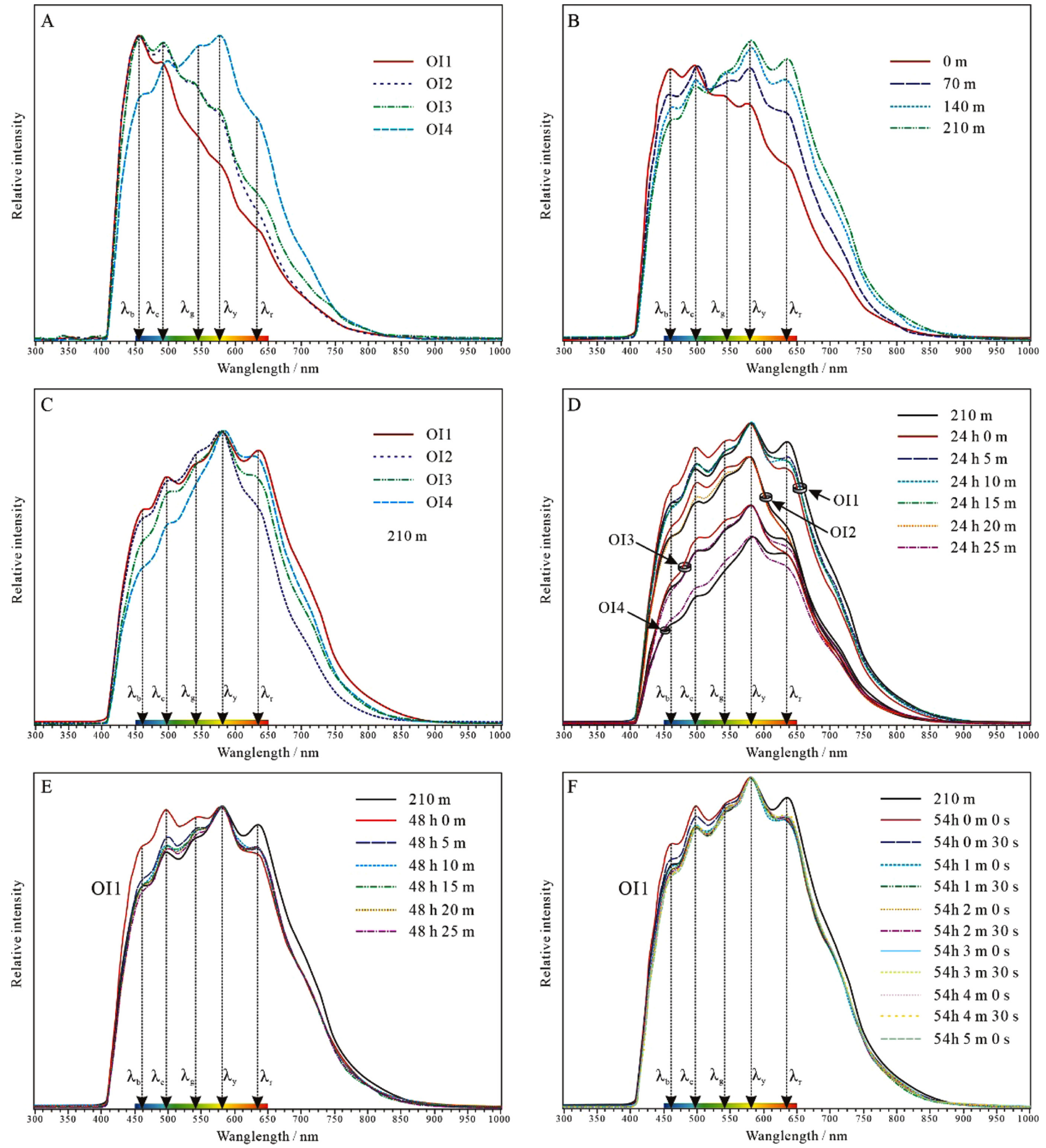

Figure 2. (A) Initial fluorescence spectra of oil inclusions. (B) Temporal fluorescence spectra of OI1. (C) Fluorescence spectra of OI1, OI2, $O I 3$ and $0 I 4$ after $U V$ irradiation for 210 minutes. (D) Comparation of spectra recorded at $210 \mathrm{~m}$ and after a 24-hour break followed by continuous UV irradiation. (E) Comparation of spectra of OI1 recorded at $210 \mathrm{~m}$ and after another 24-hour break (48 $\mathrm{h}$ later) followed by continuous UV irradiation. (F) Comparation of spectra OI1 recorded at $210 \mathrm{~m}$ and after a 6-hour break (54 h later) followed by continuous UV irradiation. Five wavelength parameters are defined as $\lambda_{b}$ (wavelength at around $455 \mathrm{~nm}$, corresponding to blue fluorescence), $\lambda_{c}$ (wavelength at around $492 \mathrm{~nm}$, corresponding to cyan fluorescence), $\lambda_{g}$ (wavelength at around $547 \mathrm{~nm}$, corresponding to green fluorescence), $\lambda_{y}$ (wavelength at around $577 \mathrm{~nm}$, corresponding to yellow fluorescence), $\lambda_{r}$ (wavelength at around $633 \mathrm{~nm}$, corresponding to red fluorescence). $h=$ hour, means how many hours have passed since the $U V$ irradiation experiment (0-210 m) started; $m$ and $s$ are abbreviations of minute and second, together represent the time length for continuous $U V$ irradiation after a certain break. 
Table 1. Initially recorded fluorescence spectral parameters of oil inclusions

\begin{tabular}{cccc}
\hline \hline Oil inclusion & $\lambda_{\max }$ & Q $_{650 / 500}$ & QF-535 \\
\hline OI1 & 456 & 0.36 & 0.87 \\
OI2 & 457 & 0.37 & 0.96 \\
OI3 & 460 & 0.44 & 1.05 \\
OI4 & 577 & 0.68 & 1.57 \\
\hline OI1 0 m & 493 & 0.61 & 1.35 \\
OI1 210 m & 582 & 1.02 & 2.09 \\
OI2 210 m & 580 & 0.77 & 1.76 \\
OI3 210 m & 581 & 0.94 & 2.08 \\
OI4 210 m & 584 & 1.20 & 2.50 \\
\hline
\end{tabular}

\section{Reversibility of Fluorescence Spectra Alteration}

The fluorescence characteristics are widely used to classify oil inclusions because it is a repeatable and cushy approach. However, previous results indicate that the UV irradiation during standard petrography study will cause fluorescence alteration of oil inclusions. To ascertain whether it is a temporary change or a permanent alteration is of great significance, because it will determine the repeatability of fluorescence study of single oil inclusion.

After a 24-hour break, a 25-minute UV irradiation was performed on the four oil inclusions. The spectra of OI1 were recorded every 5 minutes, whereas the spectra of OI2, OI3, and OI4 were recorded at two ends of UV irradiation (Fig. 2D). The spectrum of OI4 recorded at $24 \mathrm{~h} 0 \mathrm{~m}$ was damaged due to software problem.

As previously described, the UV irradiation causes a decrease in intensities of $\lambda_{\mathrm{b}}, \lambda_{\mathrm{c}}$ and $\lambda_{\mathrm{g}}$ relative to $\lambda_{\mathrm{y}}$, and an increase in intensity of $\lambda_{\mathrm{r}}$ relative to $\lambda_{\mathrm{y}}$ in both types of oil inclusions (Fig. 2C). The 24-hour break, however, caused opposite changes that the intensities of $\lambda_{b}, \lambda_{c}$ and $\lambda_{\mathrm{g}}$ increase while the intensity of $\lambda_{\mathrm{r}}$ decreases (Fig. 2D). As UV irradiation continues, the opposite alterations of $\lambda_{\mathrm{b}}, \lambda_{\mathrm{c}}$ and $\lambda_{\mathrm{g}}$ in type I oil inclusions quickly disappear and return to the shape of spectra at $210 \mathrm{~m}$. The opposite alterations of $\lambda_{\mathrm{b}}, \lambda_{\mathrm{c}}$ and $\lambda_{\mathrm{g}}$ in type II oil inclusion seem not to be easily removed by continuous UV irradiation. The spectrum of OI4 at $24 \mathrm{~h} 25 \mathrm{~m}$ is still different from the spectrum of OI 4 at $210 \mathrm{~m}$ in the relative intensities of $\lambda_{b}, \lambda_{c}$ and $\lambda_{\mathrm{g}}$. The relative intensities of $\lambda_{\mathrm{r}}$ of both types of oil inclusions also show difficulty in returning back to the position at $210 \mathrm{~m}$.

To confirm these opposite (potentially repeatable) changes and to determine how quickly they will be removed by continuous UV irradiation. Fluorescence spectra of OI1 were measured again after another 24-hour break (48h since started), and once again after a 6-hour break (54h since started) (Figs. 2E and 2F). An increase in the relative intensities of $\lambda_{\mathrm{b}}, \lambda_{\mathrm{c}}$ and $\lambda_{\mathrm{g}}$ and a decrease in the intensities of $\lambda_{\mathrm{r}}$ of the spectrum after the breaks indicates that the UV alterations on fluorescence spectra of oil inclusions are to some extent reversible. These reversible alterations were quickly removed by sharp drops in the intensities of $\lambda_{\mathrm{b}}, \lambda_{\mathrm{c}}$ and $\lambda_{\mathrm{g}}$ during the first 5 minutes (Fig. 2E), especially in the first 1 minute (Fig. 2F). However, the relative intensity of $\lambda_{\mathrm{r}}$ seems dull to the UV irradiation (Figs. 2E and F).

\section{CLSM Analysis}

A little increase in vapor bubble diameter was revealed by the comparison of two CLSM analyses before $0 \mathrm{~m}$ and after $54 \mathrm{~h} 5 \mathrm{~m}$ of the UV irradiation experiment, respectively. The diameter of vapor bubble is increased from 3.64 to $4.17 \mathrm{~mm}$. The vapor phase filling degree was increased from 8.34 to $12.54 \%$.

\section{Discussions}

\section{Validity of Fluorescence Spectra of Oil Inclusions}

Detailed petrography studies generally need to be conducted before any oil inclusion analysis. At first, researchers need to take time to find oil inclusions in thin sections under UV microscope. Second, once an oil inclusion or oil inclusion assemblage is found, more detailed observations are conducted under a range of objective lenses to determine the

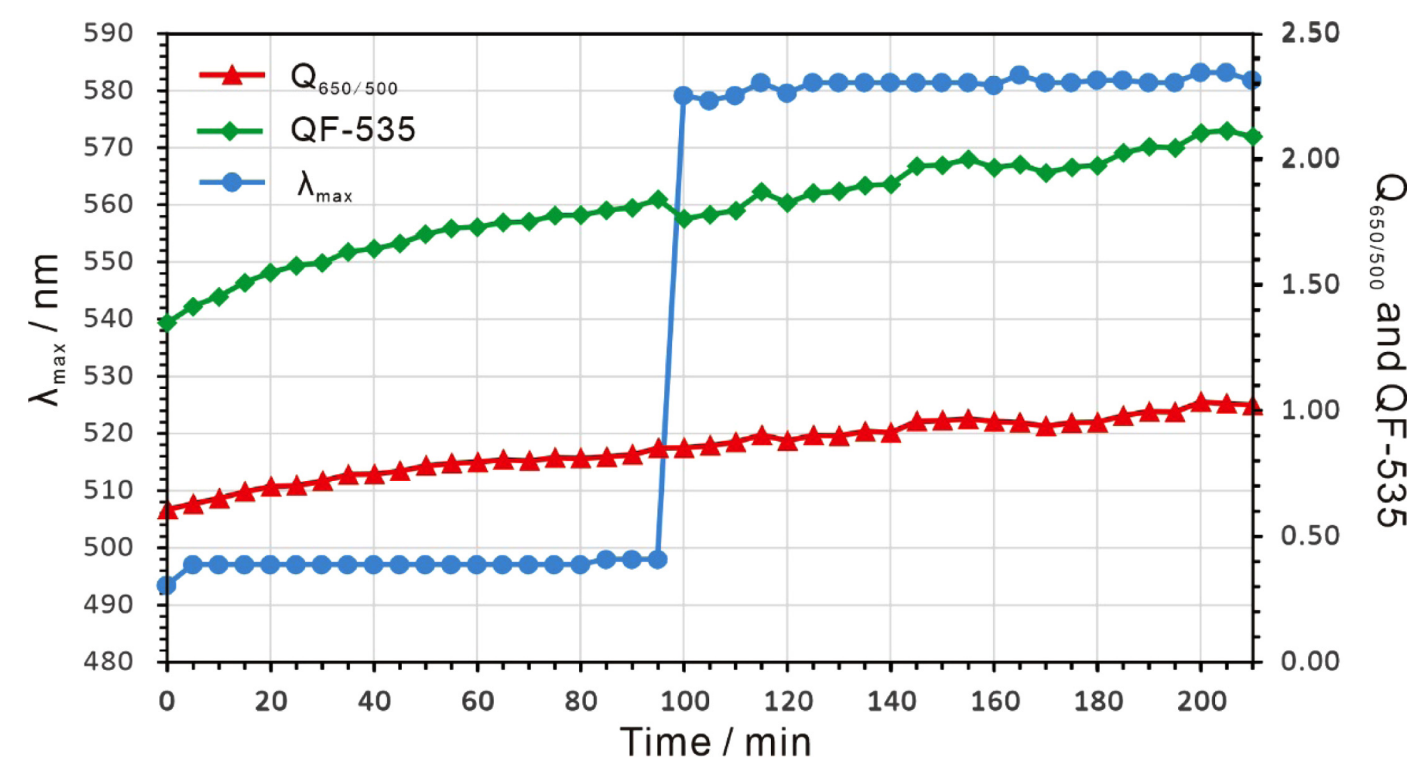

Figure 3. Temporal alteration of fluorescence spectral parameters of oil inclusion OII. 

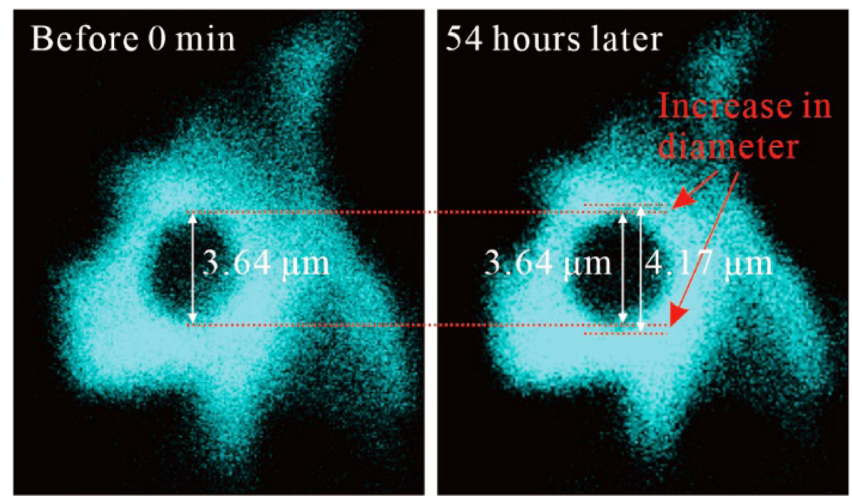

Figure 4. Photographs of OI1 under CLSM, the left one is before the $U V$ irradiation started $(0 \mathrm{~m})$, the right one is after the whole spectra analyses (54 h 5 m later).

oil inclusion origin as well as the host minerals. In the meantime, photos of the oil inclusion are taken. After that, the spectrum of an oil inclusion is measured and recorded. Hence, an oil inclusion has already experienced at least 10 minutes or more time of UV irradiation before its fluorescence spectrum is recorded. In other words, the fluorescence spectrum recorded in the routine laboratory analysis is actually an altered one.

In the UV irradiation experiment, $\lambda_{\max }$ of type I oil inclusion showed long-time stability and instant mutation, whereas both $\mathrm{Q}_{650 / 500}$ and QF535 show a continuous increase against time. The instant mutation of $\lambda_{\max }$ is considered to be caused by the relative intensity change of different peaks. The mutation of $\lambda_{\max }$ at $100 \mathrm{~m}$ shown in Fig. 3 is caused by the relative intensity of $\lambda_{\mathrm{y}}$ exceeds that of $\lambda_{\mathrm{c}}$. However, the $\lambda_{\max }$ of initial spectrum of OI 1 is $\lambda_{\mathrm{b}}$, indicating another mutation, which is caused by the relative intensity of $\lambda_{c}$ exceeding that of $\lambda_{b}$, during the CLSM analysis. Except these two mutations, $\lambda_{\max }$ stays constant. It indicates that the fluorescence spectrum shape is of significance to determine whether the $\lambda_{\max }$ is changed. If there are two peaks with similar intensities on the spectrum, the $\lambda_{\max }$ is possibly changed. For example, there are two peaks $\lambda_{1}$ and $\lambda_{2}$ with similar intensities on the spectrum and $\lambda_{\max }$ is $\lambda_{2}$. If the intensity of $\lambda_{2}$ continues to increase with UV irradiation, the original $\lambda_{\max }$ tends to be $\lambda_{1}$. However, if the intensity of $\lambda_{2}$ decreases with time, the $\lambda_{\max }$ is considered to be not changed. Although, the mutation of $\lambda_{\max }$ from one peak to another peak can be eliminated, there are still some red shifts of peaks. These red shifts generally can be ignored because the alterations are within $10 \mathrm{~nm}$. As a consequence, $\lambda_{\max }$ of fluorescence spectra recorded in laboratory routine can represent the original characteristics of oil inclusion if mutation can be eliminated by spectra shape identification; $\mathrm{Q}_{650 / 500}$ and QF-535 values recorded cannot represent the original oil inclusion because of their continuous increasing with time.

From the experiment, it can be inferred that blue fluorescence oil inclusions (type I) are more likely to be influenced by UV irradiation than yellow fluorescent oil inclusions (type II). This assumption needs to be discussed with more data in further studies to determine whether it is a common phenomenon. This experiment at least gives a warning when fluorescence study is conducted on oil inclusions, especially those with blue fluorescence. Before using the spectral parameters (mainly is $\lambda_{\max }$ ) to classify oil inclusions or to identify their thermal maturities, the validity of the spectra should be considered.

\section{Alteration of $Q_{650 / 500}$ and $Q F-535$ Values}

The alteration trends of $\mathrm{Q}_{650 / 500}$ and QF-535 in Fig. 3 show a monotonous increase and display logarithmic model. However, it is meaningless if a mathematical model is proposed without universal application. The experiment results indicate that the spectrum shape mainly alters in the first $1 \mathrm{~min}$ for the yellow spectrum $\left(\lambda_{\max }=\lambda_{y}\right)$ (Fig. 2F). For the cyan spectrum $\left(\lambda_{\max }=\lambda_{\mathrm{c}}\right.$ ) (Fig. 3), $\mathrm{Q}_{650 / 500}$ and QF-535 increase quickly in the first $20 \mathrm{~min}$, the rate of increasing gradually decreases. So, the increasing rate of $\mathrm{Q}_{650 / 500}$ and QF-535 in the first $5 \mathrm{~min}$ can be used to approximate the alteration rate before the initial spectrum is recorded. If an estimate exposure time during petrography study is obtained, the original $\mathrm{Q}_{650 / 500}$ and QF-535 values can be predicted simply by a linear calculation. For example, the spectra are assumed to be recorded from $15 \mathrm{~min}$ and the oil inclusion has experienced 15 minutes of UV irradiation. The increasing rate of $\mathrm{Q}_{650 / 500}$ between 15 and $20 \mathrm{~m}$ is $0.02 / 5 \mathrm{~min}$, the calculated $\mathrm{Q}_{650 / 500}$ at $0 \mathrm{~min}$ is 0.62 , which is very close to the measured 0.61 at $0 \mathrm{~min}$ (Table 1). The increasing rate of QF-535 between 15 and $20 \mathrm{~min}$ is 0.04 / $5 \mathrm{~min}$, the calculated QF-535 at $0 \mathrm{~min}$ is 1.39 , slightly higher than the recorded 1.35 .

The linear prediction can partially remove the increasement of $\mathrm{Q}_{650 / 500}$ and QF-535 caused by UV irradiation, however, a more precise logarithmic model is required to be established based on more experimental data, because the curves of $\mathrm{Q}_{650 / 500}$ and QF-535 over time (Fig. 3) show in logarithmic form. A precise fluorescence spectrum requires as less UV exposure time during petrography study as possible.

\section{Reversibility of UV Induced Fluorescence Alteration}

A 24-hour break can induce some opposite changes of the spectra. However, these reversible changes do not make the spectra go back to the initial shape (Figs. 2D-2F). After the CLSM analysis, even a week later, the spectrum cannot return back to the initial shape (Figs. 2A and $2 \mathrm{~B}$ ). It is inferred that the UV induced fluorescence alteration of oil inclusions is partially reversible. Once the spectrum is changed, it can hardly return to the original shape within a relatively long time (one week). As a consequence, the fluorescence spectra analysis of oil inclusions is considered to be an unrepeatable method for single oil inclusion.

\section{Potential Influence on Vapor Bubble Volume}

CLSM results show a small increase in vapor filling degree. This increase in vapor phase volume may be induced by the photo-condensation (Pradier et al., 1990). The fluorescence change to longer wavelength indicates the formation of molecules with more benzene rings (Mullins, 1998). These are the potential inner changes that may cause vapor phase volume change. However, it has been noted that the long time UV irradiation will decrease the intensity of fluorescence of oil inclusions, which will decrease the detection range of CLSM. The two runs of CLSM analyses did find a weaker image of the second run, but the signal was amplified during the second run to make the intensity of image to be the same (Fig. 4). The images, however, still show a vague difference of diameter of vapor bubbles between the two runs (Fig. 4). It is hard to make conclusions here that the vapor volume has been changed based on such slight difference of a single 
oil inclusion. The study was preliminary and considered the time consume of CLSM, so only one two-phase oil inclusion was measured vapor / liquid phase ratio by CLSM. More oil inclusions are needed in future studies.

\section{Conclusions}

Based on the UV exposure experiment of single oil inclusions, four conclusions can be draw as follows:

1) The fluorescence of oil inclusions can be significantly altered by UV irradiation during the laboratory routine petrography study. The alteration is represented by a decrease in the relative intensities of $\lambda_{b}$, $\lambda_{\mathrm{c}}$ and $\lambda_{\mathrm{g}}$, and an increase in the relative intensities of $\lambda_{\mathrm{y}}$ and $\lambda_{\mathrm{r}}$. The corresponding wavelengths of $\lambda_{b}, \lambda_{c}, \lambda_{g}, \lambda_{y}$ and $\lambda_{\mathrm{r}}$ are generally constant during UV irradiation. The $\lambda_{\max }$ shows long-time stable and instant mutation during the UV irradiation. The mutation occurs when the relative intensity of another peak (one of $\lambda_{\mathrm{b}}, \lambda_{\mathrm{c}}, \lambda_{\mathrm{g}}, \lambda_{\mathrm{y}}$ and $\lambda_{\mathrm{r}}$ ) exceeds that of the previous $\lambda_{\max }$. $\mathrm{Q}_{650 / 500}$ and QF-535 display monotonous increment during UV irradiation. The spectra of blue fluorescent oil inclusion are more likely to be altered than those of yellow fluorescent oil inclusions.

2) The original $\lambda_{\max }$ can be predicted from the altered spectra shapes. If there are two peaks with similar intensities on the spectrum, the $\lambda_{\max }$ is possibly changed from the decreasing one to the increasing one. The original $\mathrm{Q}_{650 / 500}$ and QF-535 can be predicted by using a linear calculation, in which the increasing rates of $\mathrm{Q}_{650 / 500}$ and QF-535 during the petrography study are represented by those during the first $5 \mathrm{~min}$ of spectra measurement. A more precise logarithmic model is expected to be constructed in further study with more experimental data, because the alteration $\mathrm{Q}_{650 / 500}$ and QF-535 exhibits logarithmic growth trends. A precise fluorescence spectrum requires as less UV exposure time during petrography study as possible.

3) Limited reversible changes occur after a 24-hour break. However, the spectra never return to the initial shapes within an expected time (the spectrum of OI1 even did not go back to the initial shape after a break of one week). Although, it is not confirmed whether the spectra will return to the initial shape after a longer break, a month, a year or more, in this study, it is considered that the fluorescence spectra analysis is unrepeatable within an acceptable time in laboratory routine works.

4) There is a potential change of vapor bubble volume of oil inclusions after a long time UV irradiation. More oil inclusions and more comprehensive method are needed to determine whether the vapor change is real or not; temperature measurement should be combined as they both control the PVTX modeling of oil inclusions.

\section{Acknowledgements}

This work was supported by the China National Science and Technology Major Project (2016ZX05004-001), the National Natural Science Foundation of China (No. 41730421), the Major Science and Technology Project of SINOPEC during the 13th Five-Year Plan period (No. ZDP1705) We thank Associate editor Lucia Angiolini and two anonymous reviewers for their constructive suggestions.

\section{References}

Goldstein, R.H., 2001. Fluid inclusions in sedimentary and diagenetic systems. Lithos, v. 55, pp. 159-193.

George, S.C., Ruble, T.E., and Dutkiewicz, A., 2001. The use and abuse of fluorescence colours as maturity indicators of oil in inclusions from Australasian petroleum systems. APPEA Journal, v. 41, pp. 505-522.

George, S.C., Volk, H., and Ahmed, M., 2007. Geochemical analysis techniques and geological applications of oil-bearing fluid inclusions, with some Australian case studies. Journal of Petroleum Science and Engineering, v. 57, pp. 119-138.

Munz, I.A., 2001. Petroleum inclusions in sedimentary basins: systematics, analytical methods and applications. Lithos, v. 55, pp. 195-212.

Ping, H., Chen, H., George, S.C., Li, C., and Hu, S., 2019a. Relationship between the fluorescence color of oil inclusions and thermal maturity in the Dongying Depression, Bohai Bay Basin, China: Part 1. Fluorescence evolution of oil in the context of hydrous pyrolysis experiments with increasing maturity. Marine and Petroleum Geology, v. 100, pp. $1-19$.

Ping, H., Chen, H., George, S.C., Li, C., and Hu, S., 2019b. Relationship between the fluorescence colour of oil inclusions and thermal maturity in the Dongying Depression, Bohai Bay Basin, China: Part 2. fluorescence evolution of oil in the context of petroleum generation, expulsion and cracking under geological conditions. Marine and Petroleum Geology, v. 103, pp. 306-319.

Pironon, J., and Pradier, B., 1992. Ultraviolet-fluorescence alteration of hydrocarbon fluid inclusions, Organic Geochemistry, v. 18, pp. 501509.

Pradier, B., Largeau, C., Derenne, S., Martinez, L., Bertrand, P., and Pouet, Y., 1990. Chemical basis of fluorescence alteration of crude oils and kerogens-I. Microfluorimetry of an oil and its isolated fractions; relationships with chemical structure. Organic Geochemistry, v. 16, pp. $451-460$.

Stasiuk, L.D., and Snowdon, L.R., 1997. Fluorescence micro-spectrometry of synthetic and natural hydrocarbon fluid inclusions: crude oil chemistry, density and application to petroleum migration. Applied Geochemistry, v. 12, pp. 229-241.

Mullins O.C. 1998. Optical Interrogation of Aromatic Moieties in Crude Oils and Asphaltenes. In: Mullins O.C., Sheu E.Y. (eds) Structures and Dynamics of Asphaltenes. Springer, Boston, MA. doi:10.1007/978-14899-1615-0_2 


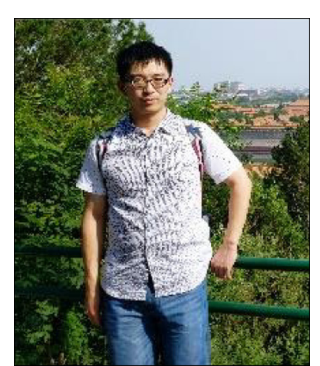

Xiuyan Liu received his bachelor's degree from China University of Geosciences (Wuhan) in 2017 and now he is a Ph.D candidate in Key Laboratory of Tectonics and Petroleum Resources of Ministry of Education, China University of Geosciences, Wuhan. His research interests are focused on hydrocarbon inclusions' PVT, petroleum accumulation, shale gas evaluation and basin modeling. He has participated in projects about the Bohai Bay Basin and Sichuan Basin in China. He has authored 3 peer-reviewed papers.

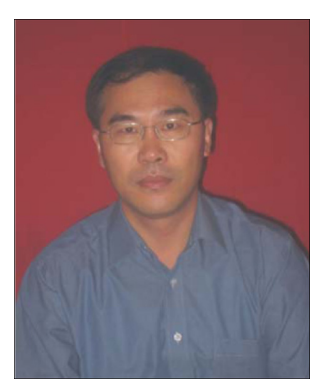

Honghan Chen is professor, doctoral supervisor in school of earth resources of China University of Geosciences(Wuhan). He received his bachelor's degree in petroleum and natural gas geology from China University of Geosciences (Wuhan) in 1985; received master's degree in petroleum from China University of Geosciences (Wuhan) in 1988; and received his Ph.D in coal, oil and gas geology from China University of Geosciences (Wuhan) in 1995. He was a research fellow at Geofluids lab in Aberdeen University in UK from 1999 to 2000. In 2005, he was named an expert on outstanding contributions of middle-aged and young people in Hubei Province. In 2006, he was hired as a "Chutian scholar" in Hubei Province. In 2007, he was selected to the Chinese Academy of Sciences "100-person program". His research interests include petroleum geology, fluid inclusions, tectonics, etc. He has authored or co-authored 128 peer-reviewed papers with 3700 citations.

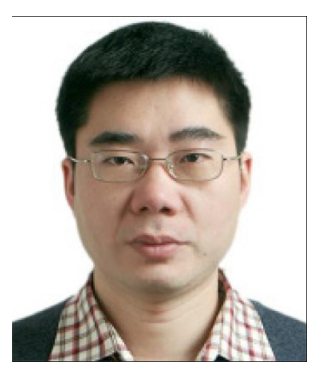

Chunquan $\boldsymbol{L i}$ was born in 1974, associate professor in China University of Geosciences (Wuhan). He received his bachelor's degree in oil and gas engineering from China University of Geosciences (Wuhan) in 1998. He received his Ph.D in mineral resource prospecting and exploration from China University of Geosciences (Wuhan) in 2003. His research interests are unconventional oil and gas accumulation mechanism, petroleum charging dynamics and systematic fluid inclusion analysis. 